\title{
A review on factors causing Parkinson's syndrome
}

\begin{abstract}
Parkinson disease (PD) is the most common chronic neurodegenerative disease that affects the brain, resulting in a progressive loss of coordination and movement to death and contributes to about $25-30 \%$ cases of dementia. An estimated seven million to 10 million people worldwide have Parkinson's disease. It is characterized by the classical motor features of parkinsonism related to Lewy bodies and loss of dopaminergic neurons in the substantia nigra, and the major symptoms include problems with resting tremor, rigidity, bradykinesia, postural instability, speech dysfunction, psychiatric symptoms, sleep disorder and fatigue. Presently existing pharmacological and surgical treatments provides relief from a few motor symptoms, but do not halt the ultimate progression of the syndrome. The root cause of Parkinson's disease remains unknown. Although significant research advances illustrate distinct paths that closely try to disclose various mitochondrial, genetic, behavioral, environmental, and epigenetic causes that may lead to the development of PD. Most likely all study target the dysfunction of the ubiquitin- proteasome pathway, alpha- synuclein protein, PINK1 protein, PI3K/Akt/mTOR pathway. However, a better understanding of the disease is leading to new diagnostic tools and treatments.
\end{abstract}

Keywords: Parkinson Disease, Dopamine, Lewybodies, Shaking Palsy, Substantia nigra.
Volume 7 Issue 4 - 2018

\author{
Suganya Selvaraj, Shanmughavel \\ Piramanayagam \\ Computational Biology Lab, Department of Bioinformatics, \\ Bharathiar University, Coimbatore, India.
}

\section{Correspondence: Shanmughavel Piramanayagam,}

Computational Biology Lab, Department of Bioinformatics, Bharathiar University, Coimbatore, India.

E-mail: shanmughavel@buc.edu.in

Received: April 28, 20I8 | Published: August 28, 2018

\section{Introduction}

Parkinson's disease (PD) was medically reported as a neurological syndrome by James Parkinson, who first explained it in "An Essay on the Shaking Palsy" in 1817. ${ }^{1}$ He illustrated this disease as started with slow, progressive involuntary tremors, followed by a complication in walking, speech, and swallowing. ${ }^{2}$ Other than motor symptoms, Parkinson's disease patients gradually experience remarkable nonmotor symptoms as well as cognition decline, sensory abnormalities, behavioral changes, fatigue, sleep disturbances, and other autonomic dysfunctions. ${ }^{3-5}$ After Alzheimer's disease (AD), PD is the most common age- related neurodegenerative disease. Although the central pathological feature of Parkinson's disease (PD) was found to be the degradation of neurons in the substantia nigra pars compacta (SNpc), (Figure 1) the loss of SNpc neurons accelerate striatal dopamine (DA) deficiency. ${ }^{6}$ Which is accountable for the key motor and nonmotor symptoms of PD.,5 PD tremor decreases the voluntary movement, so naturally, impair the daily life activities. Rigidity refers to the increased resistance (stiffness) to passive movement of a patient's limbs. Bradykinesia (very slow body movements) could considerably worsen the standared of life as a result of it takes for much longer to perform daily tasks like consumption of food or dressing, hypokinesia (reduction in movement amplitude), and akinesia (absence of normal oblivious movements, like arm swing in walking) evident as a range of symptoms, such as decreased voice volume (hypophonia), paucity of pronormal facial expression (hypomimia), drooling (inability to swallow without pondering it), diminished stride length during walking and slow writing. In addition, PD patients continuously enhance stooped attitude and will lose ordinary postural impulses, resulting in falls and, sometimes, confinement to a wheelchair. Abnormalities and cognition also occur frequently; delayed responses to queries, and slow cognitive process (bradyphrenia). ${ }^{6,7}$ Many shreds of evidence suggest that phosphatidylinositol-3 kinase (PI3K)/Akt (protein kinase-B)/mammalian target of the rapamycin (mTOR) pathway (PI3K/Akt/mTOR) pathway signaling related to dopaminergic neuron degeneration in PD. ${ }^{46-48}$ The neuron degeneration concurrently influences the wider region of the brain responsible for dementia.
In PD, dementia is not a presenting feature; it develops at least four years after PD motor symptoms start. Among PD patients, who do not primarily experience dementia the yearly occurrence varies from $2.6 \%$ to $9.6 \%$, the additive risk of dementia by the age of 85 years was over $65 \% .{ }^{8}$ Dopamine deficiency in the brain is the known causing factor of $\mathrm{PD}$, yet why this initially occurs is less clear. However, the better understanding of the pathology of Parkinson's disease is helping to identify potential drug targets for disease management.

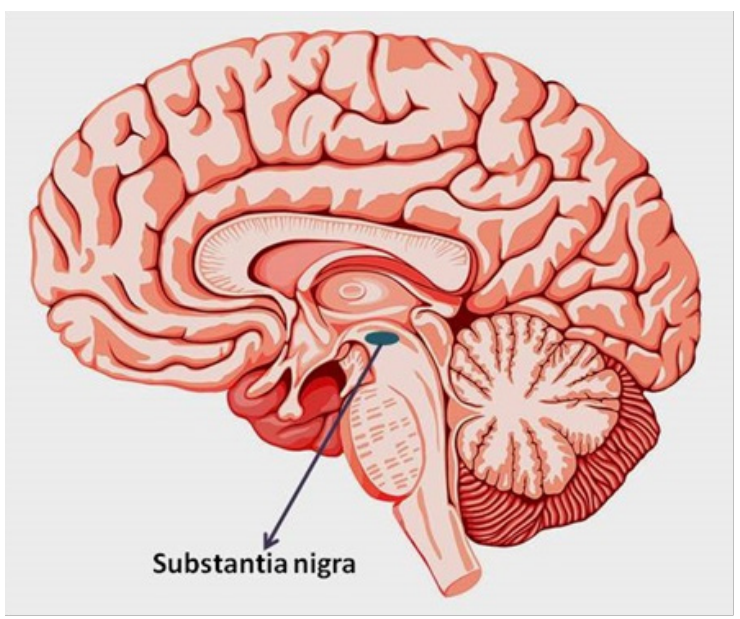

Figure I: Substantia nigra, where the dopamine degeneration occurs.

\section{Neuropathology of PD}

Degenerative parkinsonian disorder currently recognized as heterogeneous, by means of clinically considerable motor and nonmotor features. Similarly, its pathology involves wide regions of the nervous system, different neurotransmitters, ${ }^{9}$ and the most widely recognized pathologic substrates associated with anomalies within the presynaptic protein and microtubule binding protein. parkinsonian disorders constitute one among two molecular classes; tauopathies 
and $\alpha$-synucleinopathies supported pathologic aggregation of tau (microtubule binding protein) or the $\alpha$-synuclein potein (presynaptic protein) within vulnerable neurons and sometimes inside the glial cell. In idiopathic PD, accumulation of $\alpha$-synuclein in neuronal perikarya (Lewy bodies) and neuronal processes (Lewy neurites). The disease progression is multifocal and involves select peripheral autonomic sensory system neurons and central nervous system neurons. Multiple system atrophy (MSA) is another major $\alpha$-synucleinopathy. It is also conjointly linked with involuntary dysfunction and in some cases with cerebellar signs. The significant histopathologic feature of MSA is aggregation of $\alpha$-synuclein inside glial cytoplasmic inclusions (GCI). The loss of pigmentation connects with neuronal loss of dopaminergic neurons in the substantia nigra and noradrenergic neurons in the locus ceruleus. Pigment loss within the locus ceruleus is reliable with PD (Figure $2^{10}$

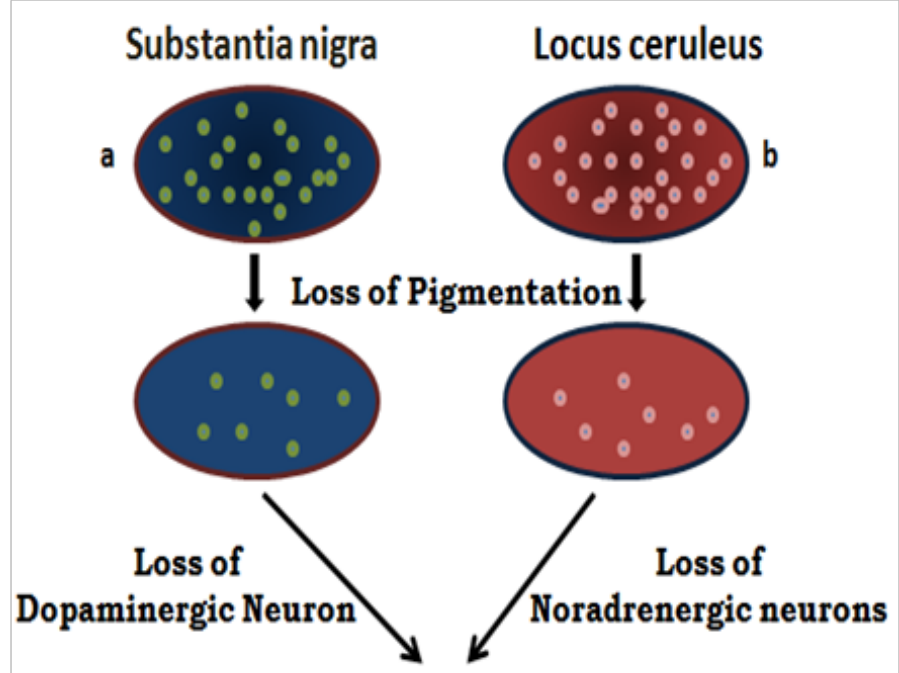

\section{PARKINSON's DisEASE}

Figure 2 Shows the Pigment loss in Substantia nigra and locus ceruleus leads Parkinson Disease.

a)Dopamine neurons in Substantia nigra

b)Noradrenergic neurons in Locus ceruleus

\section{Causes of PD}

PD involves the degeneration of dopamine neurons but it is still unknown why this dysfunction happens. However, a certain risk factor which plays a role in increasing the chances of developing PD has been identified.

\section{Age}

PD affects the population over the age of 60 (late-onset disease), while in people beyond 85 years old this prevalence reaches $5 \%$ more, featuring the effect that propelling age has on the danger of building up this condition. Although, a few numbers of patients (around 5\% of all cases) face the symptoms of PD before the age of 60years (early-onset disease) and the larger part of these cases are caused by mutations in genes.11 Early-onset cases that start before age 20 are sometimes referred to as juvenile-onset Parkinson disease.12 In PD, a neuronal loss is focused on caudal and ventrolateral regions of the
$\mathrm{SNpc}$, whereas the dorsomedial aspect of SNpc is affected throughout normal aging. 13 Thus, the process that leads age-related dopaminergic neuronal death almost certainly varies from those in PD. ${ }^{7}$

\section{Gene mutation in familial PD}

Etiology of Parkinson's disease is sporadic (not hereditarily acquired) in nature. Be that as it may, a little level of the PD patients was currently recognized to inherited gene mutations. Genes including DJ-1, ATP13A2, GIGYF2, LRRK2, HTRA2, PARK2 (parkin), SNCA, UCHL1, and PINK1 were related to autosomal dominant and recessive type of familial PD. ${ }^{4}$ Mutations in $\alpha$-synuclein gene and a missense mutation in the ubiquitin carboxy-terminal hydrolase L1 (UCHL1) gene have been identified in some familial forms of Parkinson's disease. This mutation which frequently reduces the catalytic activity of thiol protease, which responsible for the accumulation of proteins and aberrations in the proteolytic pathway. A large amount of UCH-L1 present in Lewy bodies and its contribution in the ubiquitin reliant proteolytic pathway involve it in the disease progression of Parkinson's disease, decreased enzymatic movement and improved accumulation might be in play at various phases of the sickness. The Ile93Met mutational change in the UCH-L1 gene is predicted to contribute to the genetic aetiology of just a few patients with the familial type of the illness. ${ }^{14}$

\section{Mitochondrial dysfunction}

Mitochondrial dysfunction has been concerned to be essential for the pathologic process of PD since the innovation that $\mathrm{N}-$ methyl-4-phenylpyridinium (MPP+) causes swift parkinsonism and SN cell misfortune through restraint of complex I of the electron transport chain. ${ }^{15,16}$ This identification was trailed by information of diminished complex I action and protein articulation in tissues from patients with PD. ${ }^{17,18}$ The neurons of the SN are considered to be predominant prone to dysfunction among the mitochondria and help to maintain a function of mitochondria, and particularly their dysfunction in the thrash of SN neurons in PD has been rising over late years. ${ }^{19}$ Mutational transformations in alpha-synuclein have been appeared to cause fracture of the mitochondrial system and variations in the ultra structure and allocation of the mitochondria suggesting increased and dissemination of the mitochondria proposing expanded mitochondrial fission. ${ }^{20}$ Transformations in Pink1 and Parkin have been appeared to cause a loss of mitochondrial ultra structure as observed as variation in the electron density of the mitochondria and disintegration of the mitochondrial network..$^{21,22} \mathrm{~A}$ loss of DJ-1 causes a potential degradation of mitochondrial membrane and enlarged fragmentation of mitochondria, ${ }^{23,24}$ and mitochondrial changes have additionally identified when Leucine rich repeat kinase 2 (LRRK2) is transformed. ${ }^{25,26}$ Parkin over expression has been revealed to develop the mitochondrial calcium handling capability by increasing the interaction of the mitochondria with the ER without an ensuing outcome on the mitochondrial calcium uptake machinery. ${ }^{27}$ The neurons of the $\mathrm{SN}$, utilize calcium to continue their pace making movement and in this way improvements in how the neurons handle and keep up calcium levels will be enormously powerful on both the movement and survival of these neurons. ${ }^{19}$ Familial PD gene products (LRRK2, PINK1, and DJ-1) are either localized to mitochondria or direct to a functional insufficiency in mitochondria (Parkin and DJ-1), sustaining an essential part of mitochondria in PD pathology (Figure 3)..$^{28}$ 


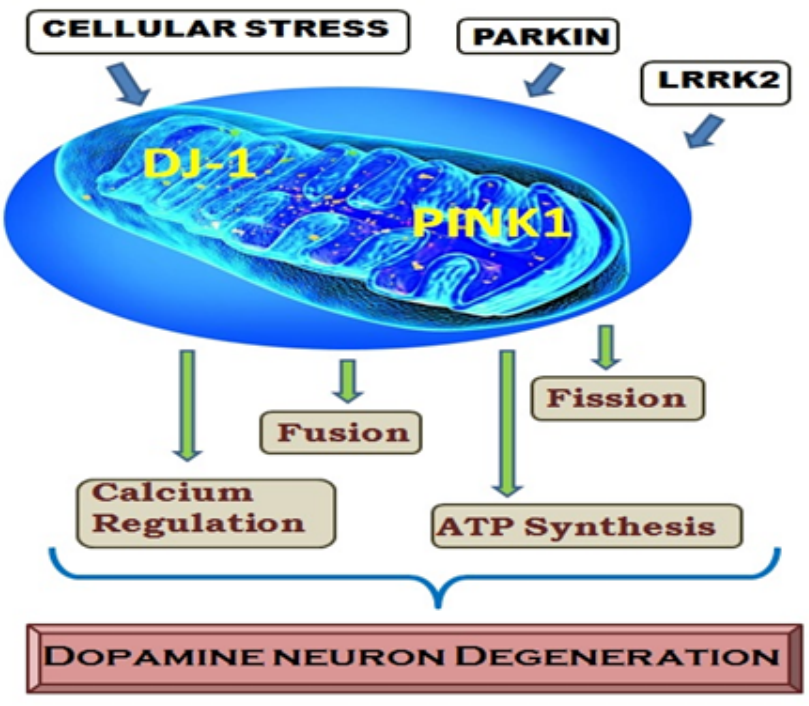

Figure $3 \mathrm{~A}$ model of mitochondria and PD pathogenesis. Cellular damage by mutations in DJ-I, PINKI, LRRK2, and PARKIN may converge on mitochondria. Identifying the specific aspects of mitochondrial dysfunction is the next challenge.

\section{miRNAs in PD}

Micro RNAs (miRNAs) contains 20-22 nucleotides (small noncoding RNA) which can control substantial arrangement of genes associated with various pathways at transcriptional levels. ${ }^{29}$ Varieties in mi RNAs and in addition mi RNA binding sites on target genes can likewise prompt PD. ${ }^{30} \mathrm{mi}$ RNA directs the levels of SNCA and DJ-1, both of which assume an essential part in PD. Expression of miR-34c and miR-34b is down regulated in the cerebrum of PD patients. Hindrance of mi R-34c and $34 \mathrm{~b}$ in neuroblastoma cells prompted a loss of mitochondrial layer potential and elevated oxidative pressure both of which are identified to biochemically connected with PD. ${ }^{31}$ When the expression ratio of $\mathrm{miR}-34 \mathrm{~b} / \mathrm{c}$ reached lower, it can direct to the accumulation of SNCA. In additionally, level of miR-34b/c decreases results in the lower expression of DJ-1 and parkin causes PD. ${ }^{32}$ Pre-clinical models deficient miR-155 showed attenuated aggravation which clarifies the contribution of miR-155 in the pathology of PD also its focal part in SNCA accumulation intervened incendiary reaction. ${ }^{33}$ Improper function in molecular chaperones such as heat shock proteins (HSP) is thought to play a pivotal function in a conglomeration of SNCA in PD. ${ }^{34,35}$ MiR-16-1 was one of the virtually screened small RNAs which had high complementarity inside the 3'-UTR of HSP-70. It was established to restrain HSP70 in cells overexpressing SNCA and indicated a circuitous direction of SNCA by raising its function when contrasted with non-transfected SH-SY5Y cells. ${ }^{36}$ In an in vitro analysis, the impact of overexpression of miR-153 and miR-7 in cortical neurons on 1-methyl-4phenylpyridinium (MPP+) initiated cell lethality was estimated and it was discovered that this progression could help in the protection of neurons from the toxicity by up regulating downstream targets of the mTOR-pathway. ${ }^{37}$ The extracellular ligand and their gene expression, projected as one of three initiators of the incorporated Parkinson's infection component, were found in the regulatory network of miRNA to be controlled by seven mi RNAs (miR-9, miR-124-1, miR-16-1, miR-128-1, miR-19a, miR-30a, and miR-23b). ${ }^{4}$ Some of those mi RNAs (miR-128-1, miR-9, and miR-124-1) have been already shown the significant role in AD neuropathogenesis being bounteously communicated in Alzheimer hippocampus. ${ }^{38}$ This may be considered as one more sign for the possible existence of common regulatory mechanisms in neurodegenerative diseases.

\section{Oxidative stress}

An oxidative stress plays a significant role in the history of PD. Expanded turnover of dopamine ought to be connected with an oxidative stress resulting from a formation of a high amount of hydrogen peroxide. While the oxidative deamination of dopamine the peroxide is formed by a mitochondrial enzyme catalyst monoamine oxidase (MAO).

Dopamine $+\mathrm{O}_{2}+\mathrm{H}_{2} \mathrm{O} \stackrel{\text { MAO }}{\rightarrow} \mathrm{H}_{2} \mathrm{O}_{2}+\mathrm{NH}_{3}+3,4$ - dihydroxyphenylacetaldehyde

Dopamine depletion and production of deaminated metabolites were correlated by a gradual rise $(87.8 \%)$ in the ratio of oxidized glutathione in brain. This modification was seen in the striatum, which is abundantly innervated by dopamine terminals, except in the frontal cortex, which obtains considerably sparser innervation by catecholamine nerve terminals. The ascent in oxidized glutathione was observed despite the fact that dopamine terminals comprise only $1 \%$ or less of the size of the striatum. Clorgyline, an inhibitor of monoamine oxidase type A, prevented the production of oxidized glutathione. These affirm that a particular elevation in neurotransmitter turnover inside nigrostriatal nerve terminals can initiate a change in cellular redox status. ${ }^{39}$

\section{Braak's hypothesis}

Braak postulates the speculation that an obscure pathogen either bacteria or virus in the gut could be a reason behind the start of sporadic $\mathrm{PD},{ }^{40}$ and they displayed a linked organizing framework for PD in view of a particular model of $\alpha$-Synuclein spreading. ${ }^{41}$ After this numerous including double hit speculation, expressing that sporadic PD initiates in two different places: the neurons in the gut and the neurons of the nasal cavity. ${ }^{42,43}$ This is currently known as Braak's theory. Which states that microbial items come into contact with olfactory and additionally enteric neurons, which stimulate the collection of $\alpha$-Synuclein? The accumulated $\alpha$-Synuclein spreads toward the focal sensory system by means of the vagus nerve and the olfactory globule. In the long run, the amassed $\alpha$-Synuclein touches base at the substantia nigra. These Genetic elements are probably going to contribute to PD, however the correct system stays to be illustrated. ${ }^{44}$ Strikingly, the theorized spread of ailment to the spinal cord just happens after the CNS has turned out to be included, thus the spinal cord isn't thought to be a potential track for the spread of PD from the periphery to the brain. ${ }^{42,45}$

\section{Conclusion}

Parkinson's disease is complex in its clinical expression and treatment. Dopaminergic degeneration is still a mystery for scientists. Many researchers directed the investigations towards pathogenesis, ${ }^{46-48}$ epidemiology, pathology, and genetics. While major research signs of progress have been made, as well as the current recognizable proof of conceivable environmental and genetic risk factors for PD, further molecular docking studies are performed to detect the molecular interactions, active site prediction, and drug interaction based studies and cellular pathway research is necessary to explicate the root causes of PD and to discover enhanced treatments. 


\section{Acknowledgments}

None.

\section{Conflict of interest}

The author declares that there is no conflict of interest.

\section{References}

1. Connolly BS, Lang AE. Pharmacological treatment of Parkinson disease: a review. JAMA. 2014;311(16):1670-1683.

2. Parkinson J. An essay on the shaking palsy 1817. Neuropsychiatry and clinical neurosciences. 2002;14:223-236.

3. Micieli G, Tosi P, Marcheselli S, et al. Autonomic dysfunction in Parkinson's disease. Neurological sciences. 2003;24(1):32-34.

4. Chandrasekaran S, Bonchev D. A Network View on Parkinson's disease. Computational and structural biotechnology journal. 2013;7(8):e201304004.

5. Ronald F, Pfeiffer. Non-motor symptoms in Parkinson's disease. Parkinsonism and Related Disorders. 2016;22(1):S119-S122.

6. Dauer W, Przedborsk S. Parkinson's Disease: Review Mechanisms and Models. Neuron. 2003;39(6):889-909.

7. Fernandez HH. Update on Parkinson disease. Cleve Clin $J$ Med. 2015;82(9):563-568.

8. Apaydin H, Ahlskog EJ, Parisi EJ, et al. Parkinsons Disease Neuropathology; Later developing dementia and loss of the Levodopa response. Arch Neurol. 2002;59(1):102-112.

9. Kalia LV, Lang AE. Parkinson's disease. Lancet. 2015;386(9996):896-912.

10.Dennis W, Dickson. Parkinson's disease and Parkinsonism: Neuropathology. Cold Spring Harb Perspect Med. 2012;2(8).

11. Amy Reeve, Eve Simcox, Doug Turnbulla. Ageing and Parkinson's disease: Why is advancing age the biggest risk factor? Ageing Res Rev. 2014;14(100):19-30.

12. Your Guide to Understanding Genetic Conditions. Genetics Home Reference; 2018.

13. Fearnley JM, Lees AJ. Ageing and Parkinson's disease: substantia nigra regional selectivity. Brain. 1991;114(5):2283-2301.

14. Leroy E, Boyer R, Auburger G, et al. The ubiquitin pathway in ParkinsonÕs disease. Nature. 1998;395(6701):451-452.

15. Langston JW, Ballard PA. Jr. Parkinson's disease in a chemist working with 1-methyl-4-phenyl-1,2,5,6-tetrahydropyridine. $N$ Engl $J$ Med. 1983;309(5):310.

16. Langston JW, Ballard P, Tetrud JW, et al. Chronic Parkinsonism in humans due to a product of meperidine-analog synthesis. Science. 1983;219(4587):979-980.

17. Schapira AH, Cooper JM, Dexter D, et al. Mitochondrial complex I deficiency in Parkinson's disease. Lancet. 1989;1(8649):1269.

18. Schapira AH, Cooper JM, Dexter D, et al. Mitochondrial complex I deficiency in Parkinson's disease. J Neurochem. 1990;54(3):823-827.

19. Reeve A, simcox E, Turnbull D. Ageing and Parkinson's disease: Why is advancing age the biggest risk factor? Ageing Res Rev. 2014;14(100):1930 .

20. Nakamura K, Nemani VM, Azarbal F, et al. Direct membrane association drives mitochondrial fission by the Parkinson disease-associated protein alpha-synuclein. J Biol Chem. 2011;286(23):20710-20726.
21. Clark IE, Dodson MW, Jiang C, et al. Drosophila pink1 is required for mitochondrial function and interacts genetically with Parkin. Nature. 2006;441(7097):1162-1166.

22. Greene JC, Whitworth AJ, Kuo I, et al. Mitochondrial pathology and apoptotic muscle degeneration in Drosophila Parkin mutants. Proc Natl Acad Sci. 2003;100(7):4078-4083.

23. Thomas KJ, Mc Coy MK, Blackinton J, et al. DJ-1 acts in parallel to the PINK1/parkin pathway to control mitochondrial function and autophagy. Hum Mol Genet. 2010;20(1):40-50.

24. Wang X, Petrie TG, Liu Y, et al. Parkinson's disease-associated DJ-1 mutations impair mitochondrial dynamics and cause mitochondrial dysfunction. J Neurochem. 2012;121(5):830-839.

25. Goo HG, Jung MK, Han SS, et al. HtrA2/Omi deficiency causes damage and mutation of mitochondrial DNA. Biochim Biophys Acta. 2013;1833(8):1866-1875.

26. Mortiboys H, Johansen KK, Aasly JO, et al. Mitochondrial impairment in patients with Parkinson disease with the G2019S mutation in LRRK2. Neurology. 2010;75(22):2017-2020.

27. Cali T, Ottolini D, Negro A, et al. alpha-Synuclein controls mitochondrial calcium homeostasis by enhancing endoplasmic reticulum-mitochondria interactions. J Biol Chem. 2012;287(22):17914-17929.

28. Li C, Beal MF. Leucine-rich repeat kinase 2: A new player with a familiar theme for Parkinson's disease pathogenesis. Proc Natl Acad Sci. 2005;102(46):16535-16536.

29. Hutvagner G, Zamore PD. A micro RNA in a multiple-turnover RNAi enzyme complex. Science. 2002;297(5589):2056-2060.

30. Singh A, Sen D. Micro RNAs in Parkinson's disease. Exp Brain Res. 2017;235(8):2359-2374.

31. Kabaria S, Choi DC, Chaudhuri AD, et al. Inhibition of miR-34b and miR-34c enhances $\alpha$-synuclein expression in Parkinson's disease. FEBS Lett. 2015;589:319-325.

32. Moyano ME, Porta S, Escaramís G, et al. Micro RNA profiling of Parkinson's disease brains identifies early down regulation of $\mathrm{miR}-34 \mathrm{~b} / \mathrm{c}$ which modulate mitochondrial function. Hum Mol Genet. 2011;20(15):3067-3078.

33. Thome AD, Harms AS, Volpicelli-Daley LA, et al. MicroRNA-155 regulates alpha-synuclein-induced inflammatory responses in models of parkinson disease. J Neurosci. 2016;36(8):2383-2390.

34. Auluck PK, Chan HYE, Trojanowski JQ, et al. Chaperone suppression of alpha-synuclein toxicity in a Drosophila model for Parkinson's disease. Science. 2002;295(5556):865-868.

35. Klucken J, Shin Y, Masliah E, et al. Hsp70 reduces alpha-synuclein aggregation and toxicity. J Biol Chem. 2004;279(24):25497-25502.

36.Zhang Z, Cheng Y. miR-16-1 promotes the aberrant $\alpha$-synuclein accumulation in Parkinson disease via targeting heat shock protein 70. Sci World J. 2014:1-8.

37. Fragkouli A, Doxakis E. miR-7 and miR-153 protect neurons against MPP(+)-induced cell death via upregulation of mTOR pathway. Front Cell Neurosci. 2014;8:182.

38. Lukiw WJ. Micro-RNA speciation in fetal, adult and Alzheimer's disease hippocampus. Neuroreport. 2007;18(3):297-300.

39. Spina BM, Cohen G. Dopamine turnover and glutathione oxidation: Implications for Parkinson disease. Proc Natl Acad Sci. 1989;86(4):1398-1400.

40. Braak H, Rub U, Gai WP, et al. Idiopathic Parkinson's disease: possible routes by which vulnerable neuronal types may be subject to neuroinvasion by an unknown pathogen. J Neural Transm. 2003;110(5):517-536. 
41. Braak H, Tredici DK, Rüb U, et al. Staging of brain pathology related to sporadic Parkinson's disease. Neurobiol Aging. 2003;24(2):197-211.

42. Hawkes CH, Del Tredici K, Braak H. Parkinson's disease: a dual-hit hypothesis. Neuropathol Appl Neurobiol. 2007;33(6):599-614.

43. Hawkes CH, Del Tredici K, Braak H. Parkinson's disease: the dual hit theory revisited. Ann NY Acad Sci. 2009;1170:615-22.

44. Rietdijk CD, Pardo PP, Garssen J, et al. Exploring Braak's Hypothesis of Parkinson's Disease. Front Neurol. 2017;8:1-37.

45. Del Tredici K, Braak H. Review: sporadic Parkinson's disease: development and distribution of $\alpha$-synuclein pathology. Neuropathol Appl Neurobiol. 2016;42(1):33-50.

46. Giacoppo S, Bramanti P, Mazzon E. Triggering of inflammasome by impaired autophagy in response to acute experimental Parkinson's disease: involvement of the PI3K/Akt/mTOR pathway. Neuroreport. 2017;28(15):996-1007.

47. Wang G, Pan J, Chen SD. Kinases and kinase signaling pathways: potential therapeutic targets in Parkinson's disease. Prog Neurobiol. 2012;98(2):207-221.

48. Khwanraj K, Madlah S, Grataitong K, et al. Comparative mRNA expression of eEF1A isoforms and a PI3K/Akt/m TOR Pathway in a cellular model of Parkinson's disease. Parkinsons Dis. 2016;2016:1-11. 